\title{
Green Pigmentation of Teeth Caused by Neonatal Cholestatic Jaundice and Sepsis: A Case Report
}

\author{
Gagandeep Lamba ${ }^{1}$, Devendra I Nagpal ${ }^{2}$, Roopa Korishettar ${ }^{3}$
}

\begin{abstract}
Aim: The aim of this article is to report a case of green discoloration of primary teeth associated with neonatal cholestatic jaundice and sepsis in a 2-year-old girl.

Background: Systemic changes can lead to dental alterations of the teeth. One of the disorders is elevated serum bilirubin levels that can cause pigmentation of teeth. Green pigmentation of teeth is uncommon, but when it occurs, is a cause of concern for parents and the child, as he may also loose self-confidence.

Case description: The parents reported to the clinic with the child whose chief complaint was green pigmented teeth. Her medical records revealed she was preterm and was diagnosed with neonatal cholestatic jaundice which lead to hyperbilirubinemia and sepsis. On review at 36 months of age, all her deciduous teeth were completely erupted and a striking demarcation was visible between the green incisal edges and the normally colored cervical one-thirds of all primary teeth. Treatment alternatives were suggested and the child was kept on regular follow-ups. Conclusion: So, one should be aware of such condition and seek medical practitioner or reports to confirm the diagnosis. If such a condition exists, esthetic treatment can be achieved with the newer techniques in cosmetic dentistry.

Clinical significance: Green pigmentation has very limited causes. The time of illness coincides with the stage of tooth formation. Proper medical history is important to evaluate the extent of pigmentation and probable involvement of the permanent teeth.

Keywords: Green teeth, Hyperbilirubinemia, Jaundice, Neonatal jaundice, Pigmented teeth, Tooth discoloration.

International Journal of Clinical Pediatric Dentistry (2019): 10.5005/jp-journals-10005-1696
\end{abstract}

\section{BACKGROUND}

A wide array of reasons causes discoloration of deciduous teeth. Based on the location of the stains, discoloration can be classified as intrinsic or extrinsic. ' Extrinsic stains can be due to dental plaque, calculus, food and beverages, tobacco, chromogenic bacteria, metallic compounds, and topical medications. ${ }^{2}$ These stains can be removed by polishing with a rubber cup and appropriate polishing paste.

Intrinsic discoloration is due to incorporation of the stain in enamel and dentin either during the formation of teeth or after the formation is complete. ${ }^{3}$ Some of the causes of intrinsic discoloration include dental restorative materials (silver amalgam), dental traumatic injuries, enamel hypoplasia infection of succedaneous tooth (Turner's tooth), medications like tetracycline, and developmental disturbances like amelogenesis imperfecta, dentinogenesis imperfecta, and dentin dysplasia. Nutritional deficiency; metabolic disorders like excess of fluoride, erythropoietic porphyria, and alkaptouria; ${ }^{4}$ and systemic diseases like sickle cell anemia, thalassemia, hemolytic disease of newborn due to $\mathrm{Rh}$ incompatibility, and biliary atresia cause hyperbilirubinemia and subsequently dose-dependent deposition of bilirubin throughout the body. Bilirubin disappears from the soft tissues following remission of hyperbilirubinemia. However, in hard tissues bilirubin gets trapped permanently causing green discoloration of teeth. ${ }^{5}$

This article reports a case of green discoloration of primary teeth associated with neonatal cholestatic jaundice caused by sepsis.

\section{Case Description}

A 2-year-old Indian girl was referred to the general dentistry clinic of Prestos Hospital of Nagpur for evaluation of green discoloration

\footnotetext{
1,2 Department of Pediatric and Preventive Dentistry, VSPM's Dental College and Research Centre, Nagpur, Maharashtra, India

${ }^{3}$ Department of Pedodontics, College of Dental Sciences, Davangere,
} Karnataka, India

Corresponding Author: Gagandeep Lamba, Department of Pediatric and Preventive Dentistry, VSPM's Dental College and Research Centre, Nagpur, Maharashtra, India, Phone: +91 8983184861, e-mail: drgagandeeplamba@gmail.com

How to cite this article: Lamba G, Nagpal DI, Korishettar R. Green Pigmentation of Teeth Caused by Neonatal Cholestatic Jaundice and Sepsis: A Case Report. Int J Clin Pediatr Dent 2019;12(6):566-568.

\section{Source of support: Nil}

Conflict of interest: None

of her teeth. Her medical records revealed that she was a preterm baby born at 30 weeks gestation with a low birth weight of $1.5 \mathrm{~kg}$. She had patent ductus arteriosus at birth which was closed after single dose of ibuprofen. She also had electrolyte imbalance and received intensive treatment for several months after birth. She had signs of respiratory distress for which she was kept on ventilator. She also had neonatal enterocolitis for which she was kept nil by mouth (NBM), and she had generalized edema for which she was given diuretics. Patient was severely jaundiced and her bilirubin levels increased from $29.8 \mathrm{mg} / \mathrm{dL}$ to $32.3 \mathrm{mg} / \mathrm{dL}$ within few days. She was diagnosed with neonatal cholestatic jaundice and neonatal sepsis with Staphylococcus aureus. On discharge, her bilirubin levels were $19.5 \mathrm{mg} / \mathrm{dL}$ and this further reduced to normal levels at approximately 4 months after birth.

The initial clinical examination of green teeth revealed completely erupted upper and lower primary incisors, erupting 


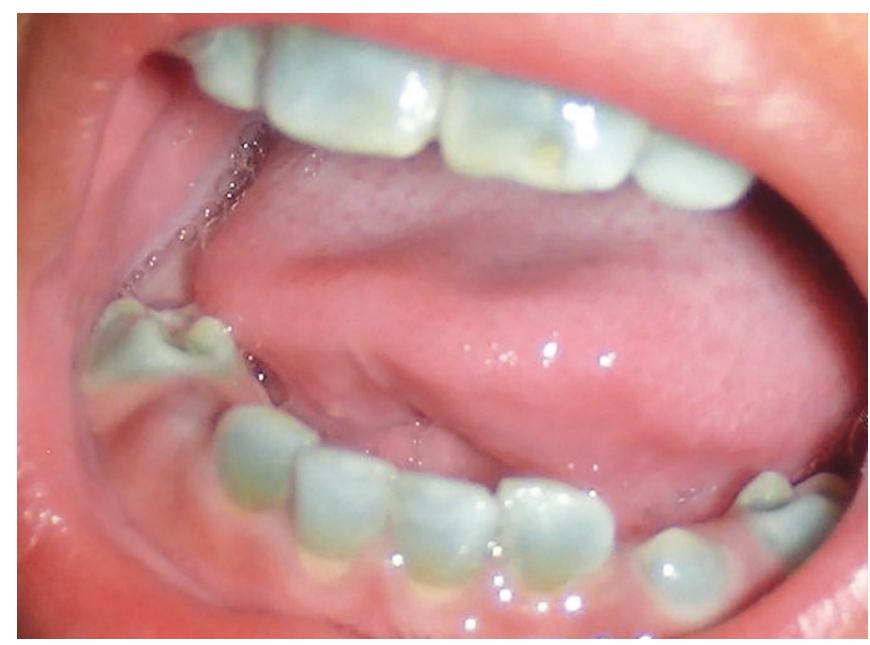

Fig. 1: Distinct green discoloration of incisal and middle one-third of the crowns of primary incisors

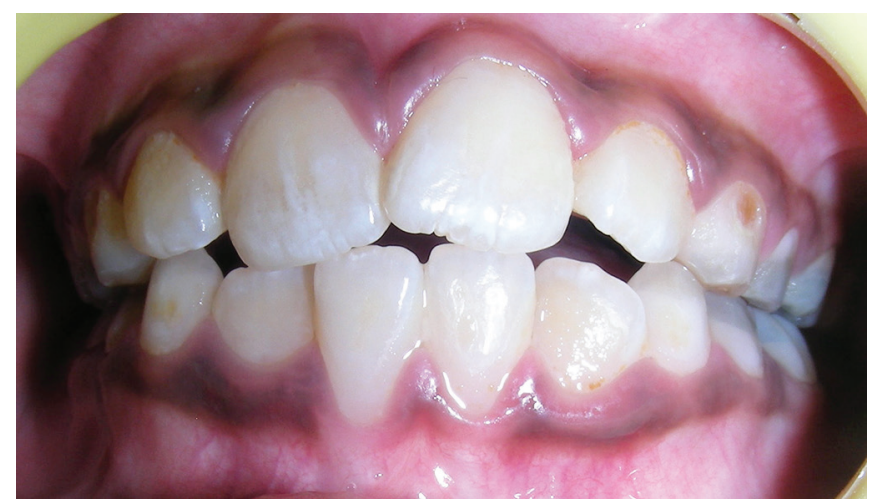

Fig. 3: No discoloration of permanent incisors

mandibular canines, and first molars. The clinical portion of incisors revealed a distinct green discoloration of incisal and middle onethird of the crowns in contrast to the normally colored cervical one-third (Fig. 1). The child's mouth was otherwise exceptionally clean; the oral soft tissues were healthy, pink, and normal. Differential diagnosis included the following:

- Extrinsic stains caused due to chromogenic bacteria.

- Dentinogenesis imperfecta.

- Amelogenesis imperfecta.

- Rh incompatibility.

Based on the history, clinical findings, and investigations, we came to the final diagnosis of green pigmentation due to hyperbilirubinemia.

Parents were explained about poor esthetics due to green pigmentation and dental management for the same. They were also informed about probable involvement of permanent teeth. But as the patient was very young, the parents did not agree for any treatment at this stage. So they were explained the importance of oral hygiene and was kept on regular follow-ups.

On a follow-up visit, at thirty-six months of age, all her primary teeth were completely erupted and a striking demarcation was visible between the green incisal thirds and cusp tips and the normally colored cervical one-third of the clinical crown. A significant finding that was observed was that the greenish

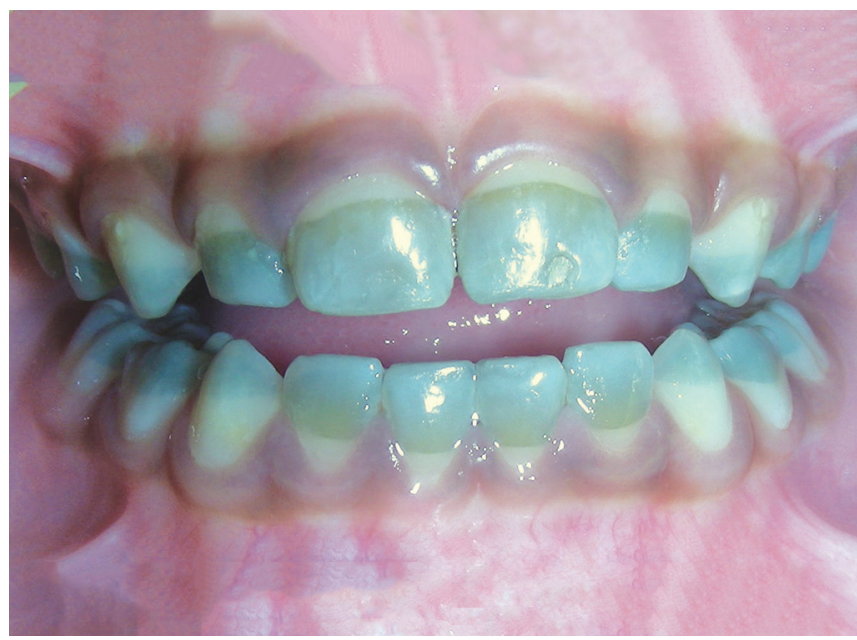

Fig. 2: Green discoloration covering most of the crown structure of primary incisors while the discoloration is only on incisal thirds in canines and reduced to only cuspal region in molars

discoloration covered most of the crown structure of incisors while the discoloration reduced to only the incisal thirds in canines and was further reduced to the cuspal region in primary molars (Fig. 2). The child continued to display good oral hygiene and healthy soft oral tissues.

The child reported for oral health checkup at 7 years of age. Intraoral findings revealed completely erupted permanent upper and lower incisors and first molars. These teeth did not display green pigmentation (Fig. 3).

\section{Discussion}

When a child presents with discolored teeth, one needs to decide whether the discoloration is in enamel or dentin, whether it is associated with other problems and whether the discoloration is genetic or environmental in etiology. ${ }^{6}$ Bilirubin is a degradation product of hemoglobin. This unconjugated bilirubin is converted to conjugated bilirubin in liver and eventually excreted from the body through urine as urobilin and stools as stercobilin. This process is highly efficient under normal conditions. Any disturbance in this pathway can lead to elevated levels of bilirubin in blood or hyperbilirubinemia.

Normal plasma bilirubin levels are-0.3-1 mg/dL. Hyperbilirubinemia is when bilirubin levels increase to $>1.5 \mathrm{mg} / 100 \mathrm{~mL}$. Green discoloration is generally seen when bilirubin levels are $>30 \mathrm{mg} / \mathrm{dL}^{7}$

In our case, bilirubin levels were $32.5 \mathrm{mg} / \mathrm{dL}$ immediately after birth, which could be a causative factor for green pigmentation. As hard tissues lose their metabolic activity after maturation, bilirubin is permanently trapped in teeth leading to intrinsic stains. On the contrary, even soft tissues are pigmented but due to their high turnover rate, pigmentation disappears over a period of time. ${ }^{8}$

The etiology of neonatal hyperbilirubinemia is varied during neonatal period. Various causes of neonatal hyperbilirubinemia include premature birth, respiratory distress syndrome, extravasation of blood, and congenital hypothyroidism.

Respiratory distress causes neonatal hyperbilirubinemia by keeping ductus venosus functionally patent and thereby causing poor perfusion of hepatic sinusoids and decreasing bilirubin excretion. Respiratory distress was seen in our case also. 
Extravasation of blood leads to shortening of life span of erythrocytes. Macrophages quickly engulf these erythrocyte hemoglobin and convert them to bilirubin.

In our case also, it was a preterm birth and child exhibited both respiratory distress and extravasation of blood, further contributing to hyperbilirubinemia. ${ }^{2,9}$

Green pigmentation is seen from the translucent enamel and is the result of reaction of developing tooth to the bilirubin-rich blood. In the past, it was believed that the green color is basically the oxidation product of bilirubin called biliverdin. ${ }^{9}$ However, a study done by Shibata et al. proved that the green color comes from the accumulation of bilirubin and not of biliverdin. It is known that the location and extent of green pigmentation coincides with the period of tooth formation. ${ }^{10}$ The calcification process of primary teeth begins during the 4th month of fetal life and ends almost at the 11th month after birth with primary second molars. The dental crown of incisors are completely formed 1 month after birth and molars and canines by 6th month after birth. In our case, hyperbilirubinemia was at its peak for first 3-4 month after birth, which coincide with calcification of primary teeth and hence pigmentation was significant in incisors. As the bilirubin levels reduced, the pigmentation was evident only in occlusal one-third of molars and incisal tips of canines, which would have completed after birth. ${ }^{9}$ As calcification of permanent teeth has its beginning from the birth, the possibility of pigmentation of permanent teeth and especially the first permanent molars could be a possibility. Hence, the patient was kept on a regular follow-up. But, the permanent first molars did not show any signs of green pigmentation.

Differential diagnosis of green teeth includes the following: extrinsic discoloration caused due to chromogenic bacteria and medicaments; and intrinsic discoloration caused due to dentinogenesis imperfecta, amelogenesis imperfecta, tetracycline, and congenital erythropoietic porphyria. ${ }^{11}$ The final diagnosis of bilirubin pigmentation was based on postnatal history of jaundice combined with green discoloration and negligible enamel hypoplasia. ${ }^{11}$ To date only one case reported by Guimaraes et al. has been reported in which green teeth were associated with Klebsiella and Candida. ${ }^{11}$ Herbst et al. described a case where the patient had staphylococcal sepsis but did not correlate it with jaundice. Our case also had staphylococcal sepsis but no correlation could be found with green pigmentation.

Green pigmentation can cause anxiety to parents about esthetics and possible discoloration of permanent dentition. To make a decision about esthetic treatment, it is important to evaluate whether the pigmentation is in dentin and/or enamel and it is related to other problems. ${ }^{9}$ In our case, there was no family history of the pigmentation, suggesting the discoloration could be due to systemic imbalance occurring after birth.

Knowledge of chronological eruption of teeth and timing and severity of hyperbilirubinemia will help the clinician determine the probability of pigmentation in permanent teeth. As it is intrinsic staining, restorative materials do not cover the pigmentation completely, and hence, esthetic treatment is required to improve the normal physical, psychological, and social development of the patient.

Cosmetic treatment becomes important as the child grows older and attends school; the presence of discolored teeth can cause peer problems. ${ }^{12}$ Thus, cosmetic treatment becomes priority to improve self-esteem and assist social acceptance. ${ }^{11}$

Treatment options include composites, resin restorations, as well as strip crowns for anterior teeth. In our case, as the parents were not willing for the treatment, no treatment was done but the patient was kept on a regular follow-up. The parents were also counseled about the various problems the child might face due to peer teasing, which may hamper her social interaction and hence self-esteem.

\section{Conclusion}

Today, as a result of advanced technology, green teeth are considered a rarity. When parents see green teeth for the first time, they often are anxious and consult a dentist. So, one should be aware of such condition and seek medical practitioner or reports to confirm the diagnosis. If such a condition exists, esthetic treatment can be achieved with the newer techniques in cosmetic dentistry. Esthetic treatment should be considered primarily to prevent peer teasing and build confidence of the child. As pediatric dentists, we hold a better position to diagnose, counsel, and treat such children.

\section{Clinical Significance}

This clinical case adds to the literature a rare entity which has a strong medical background and hence emphasizing the importance of taking thorough medical history. This is important to dentist as it affects the teeth to a large extent. The etiological factors, clinical history, and management have been dealt in detail in this article. This article is unique as it was followed for a long period of time, i.e., till the eruption of permanent incisors and molars.

\section{References}

1. Watts A, Addy M. Tooth discolouration and staining: a review of the literature. Br Dent J 2001;190(6):309-316. DOI: 10.1038/sj.bdj. 4800959

2. Herbert FL, Delcambre TJ. Unusual case of green teeth resulting from neonatal hyperbilirubinemia. ASDC J Dent Child 1987;54(1):54-56.

3. Hattab FN, Qudeimat MA, Al-Rimawi HS. Dental discoloration: an overview. J Esthet Dent 1999;11(6):291-310. DOI: 10.1111/j.17088240.1999.tb00413.x.

4. Culpepper WD. A comparative study of shade-matching procedures. J Prosthet Dent 1970;24(2):166-173. DOI: 10.1016/0022-3913(70) 90140-X.

5. Watanabe K, Shibata T, Kurosawa T, et al. Bilirubin pigmentation of human teeth caused by hyperbilirubinemia. J Oral Pathol Med 1999;28(3):128-130. DOI: 10.1111/j.1600-0714.1999.tb02010.x.

6. Barta JE, King DL, Jorgensen RL. ABO blood group incompatibility and primary tooth discoloration. Pediat Dent 1989;11(4):316-318.

7. Amaral TH, Guerra C, Bombonato-Prado K, et al. Tooth pigmentation caused by bilirubin: a case report and histological evaluation. Spec Care Dentist 2008;28(6):254-257. DOI: 10.1111/j.17544505.2008.00048.x.

8. Naudi AB, Ammari AB, Fung DE. A report of 2 cases of green pigmentation in the primary dentition associated with cholestasis caused by sepsis. J Dent Child 2008;75(1):91-94.

9. Alto LA, Pomarico L, Souza IP, et al. Green pigmentation of deciduous teeth: report of two cases. J Dent Child 2004;71(2):179-182.

10. Shibata T, Watanabe $\mathrm{K}, \mathrm{Oda} \mathrm{H}$, et al. Experimental bilirubin pigmentation of rat dentine and its detection by a qualitative analytical method. Arch Oral Biol 1996;41(5):509-511. DOI: 10.1016/ 0003-9969(95)00145-x

11. Guimaraes LP, Silva TA. Green teeth associated with cholestasis caused by sepsis: a case report and review of the literature. Oral Surg Oral Med Oral Pathol Oral Radiol Endod 2003;95(4):446-451. DOI: 10.1067/moe.2003.43.

12. Rosenthal $P$, Ramos A, Mungo R. Management of children with hyperbilirubinemia and green teeth. J Pediatr 1986;108:103-105. DOI: 10.1016/S0022-3476(86)80780-6. 